\title{
STATUS OF THE INTERNATIONAL MUON IONIZATION COOLING EXPERIMENT (MICE) ${ }^{\dagger}$
}

\author{
Michael S. Zisman , LBNL, Berkeley, CA 94720, U.S.A. \\ for the MICE Collaboration
}

\begin{abstract}
An international experiment to demonstrate muon ionization cooling is scheduled for beam at Rutherford Appleton Laboratory (RAL) in 2007. The experiment comprises one cell of the Study II cooling channel [1], along with upstream and downstream detectors to identify individual muons and measure their initial and final 6D phase-space parameters to a precision of $0.1 \%$. Magnetic design of the beam line and cooling channel are complete and portions are under construction. The experiment will be described, including cooling channel hardware designs, fabrication status, and running plans. Phase 1 of the experiment will prepare the beam line and provide detector systems, including time-of-flight, Cherenkov, scintillating-fiber trackers and their spectrometer solenoids, and an electromagnetic calorimeter. The Phase 2 system will add the cooling channel components, including liquid-hydrogen absorbers embedded in superconducting Focus Coil solenoids, 201-MHz normalconducting RF cavities, and their surrounding Coupling Coil solenoids. The MICE Collaboration goal is to complete the experiment by 2010; progress toward this is discussed.
\end{abstract}

\section{INTRODUCTION}

There has been considerable interest in recent years in the design of a muon storage ring facility to provide an intense beam of neutrinos for very long baseline (3000$7500 \mathrm{~km}$ ) experiments. Such experiments would permit detailed study of neutrino oscillations, would determine the neutrino mass hierarchy, and would probe whether neutrinos exhibit the phenomenon of charge-conjugationparity (CP) violation. Discovery of CP violation in the lepton sector would provide supporting evidence that the observed baryon asymmetry in the Universe - the fact that we live in a matter-dominated Universe-is due to leptogenesis. Understanding why any matter survived after the Big Bang is clearly one of the most fundamental unanswered questions in particle physics today.

In the majority of Neutrino Factory designs undertaken to date (see Ref. [1] and references contained therein), the facility has included an ionization cooling section. Ionization cooling of muons reduces their transverse phase space sufficiently to permit subsequent acceleration and storage in a practical acceleration system. The need for ionization cooling results from the production method of the muons, that is, from the fact that they arise from the decays of secondary pions produced by proton

\footnotetext{
This work was supported by the Office of Science, U. S. Department of Energy, under Contract No. DE-AC02-05CH11231.

"mszisman@1bl.gov
}

interactions with a target material. Muons are thus a tertiary beam with a very large transverse phase space and a large energy spread as well. While several techniques for cooling beams have been developed, only ionization cooling is rapid enough to be practical for cooling muons, which are unstable particles with a lifetime at rest of $2.2 \mu \mathrm{s}$.

The international Muon Ionization Cooling Experiment, (MICE, see http://www.mice.iit.edu/), is designed to test one cell of a cooling channel lattice under various conditions to demonstrate our understanding of the muon cooling process. Implementing such a system will tell us about the subtleties of fabrication of the required components and, after comparing our results with detailed simulation predictions, will provide a validated tool for designing and optimizing the performance of a future Neutrino Factory cooling channel. A Muon Collider has even more stringent requirements on cooling, so its design will benefit greatly from our results.

\section{Ionization Cooling}

The ionization cooling process is conceptually straightforward, though its implementation is rather challenging. The basic principle is illustrated in Fig. 1. We employ 201-MHz RF cavities to obtain sufficient aperture at a respectable gradient (nominally $16 \mathrm{MV} / \mathrm{m}$ ). The absorber of choice is liquid hydrogen, though other low- $Z$ materials, such as $\mathrm{LiH}$, are considered viable alternatives, at least in the Neutrino Factory parameter regime.

The implementation of an ionization cooling scheme is very complicated, requiring high-power RF cavities in close proximity to liquid hydrogen. The layout employed for MICE is shown in Fig. 2. Adding the RF power feeds and tuners for the cavities, safety systems for the liquidhydrogen, and vacuum systems makes the region very crowded.

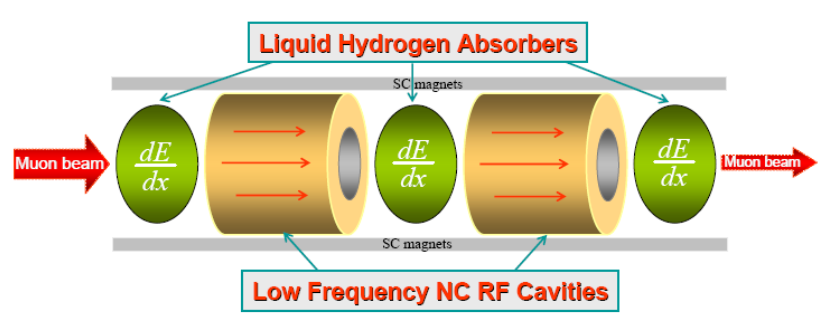

Figure 1: Schematic of ionization cooling concept. Ionization energy loss in all three planes occurs in absorbers, whereas the RF cavities restore only the longitudinal component. Repeating the process many times reduces $p_{x, y}$ with respect to $p_{z}$, and thus reduces the transverse emittance. The channel is immersed in superconducting solenoids to maintain the beam focusing. 


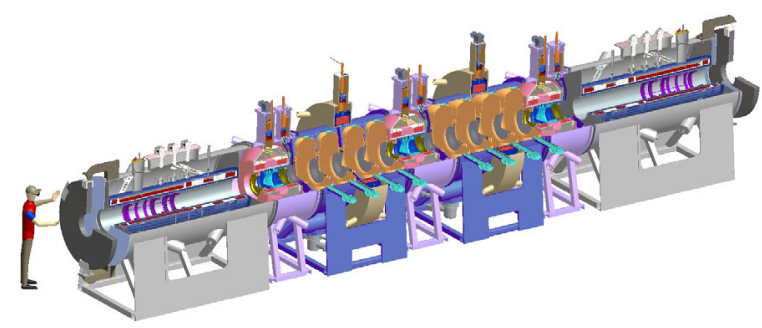

Figure 2: Cutaway view of the MICE cooling channel. Spectrometer solenoids upstream and downstream bracket the cooling channel hardware.

\section{MUON BEAM LINE}

Although there was previously a muon beam line at RAL, it needed extensive modification to be suitable for MICE. The secondary beam line is therefore being entirely rebuilt. The line will provide beams of $140-240 \mathrm{MeV} / \mathrm{c}$ muons, with normalized emittance values in the range of $1-10 \pi \mathrm{mm}-\mathrm{rad}$. Magnets for the line are presently being refurbished and remeasured, and those located within the ISIS shielded enclosure are being installed this summer. Figure 3 shows the layout of the new muon beam line, which comprises three quadrupole triplets, two dipoles, a lead diffuser to create the required incoming emittance, and a 5-m long decay solenoid that was provided by Paul Scherrer Institute. Unfortunately, the decay solenoid has recently developed a leak in the high-pressure He system, which will likely delay installation until the next ISIS shutdown (planned for December 2007).

The plunging target mechanism (Fig. 4) was completely remade and installed in ISIS for testing. An issue with wear of the bearings has been found and is under study.

\section{COOLING CHANNEL}

The cooling channel is the heart of MICE. It comprises three absorbers [2] located in three Focus Coil modules [3], and two RF-Coupling-Coil (RFCC) modules [4].

Each hydrogen absorber contains about $20 \mathrm{~L}$ of liquid. Aluminum beam windows are $300 \mathrm{~mm}$ diameter with a

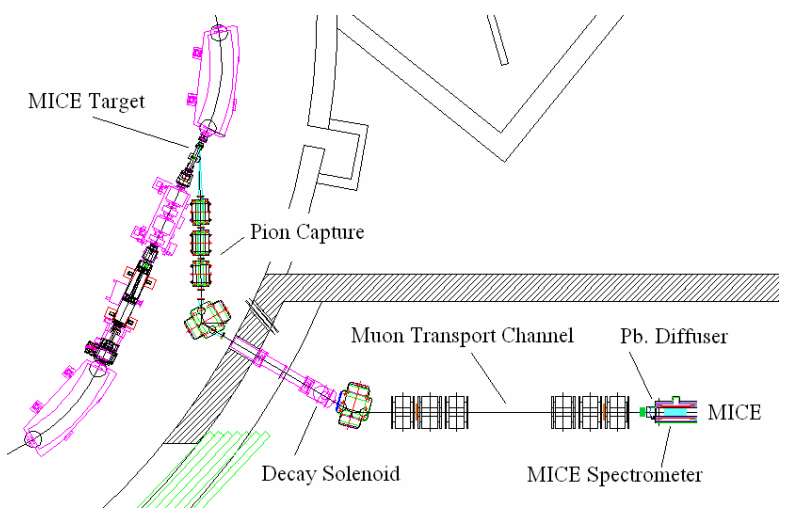

Figure 3: Layout of muon beam line at RAL. The target is installed in the $800 \mathrm{MeV}$ ISIS proton storage ring.

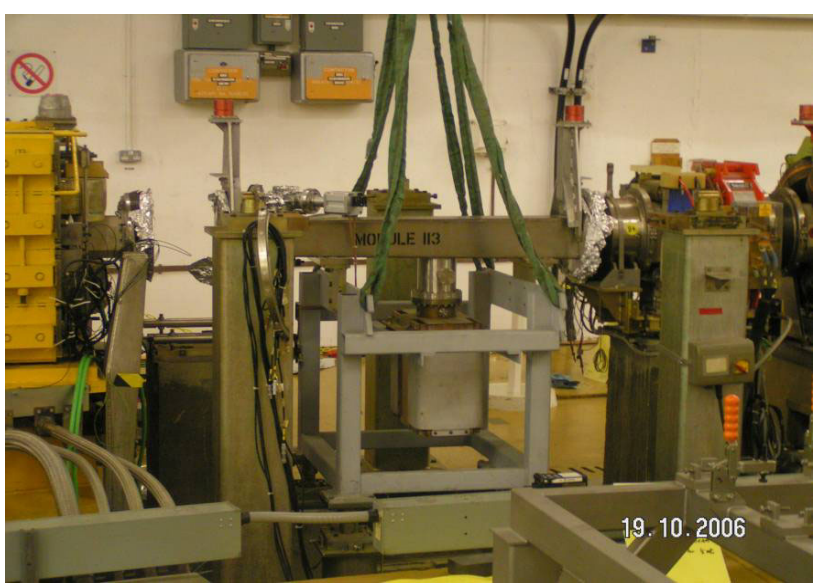

Figure 4: Plunging target mechanism in the ISIS ring.

thickness of about $150 \mu \mathrm{m}$. Each absorber is contained within a pair of room temperature safety windows (see Fig. 5), with parameters identical to those of the absorber windows. Figure 5 also shows the Focus Coil module into which the absorber is inserted. There are two focus coils that can be powered either with opposite polarity or the same polarity. Both configurations will be tested. Cryocoolers will be used for cooling the magnet coils.

The eight 201-MHz RF pillbox cavities will be very similar to the prototype cavity [5] already under test in the MuCool Test Area (MTA) at Fermilab. Each will have beryllium windows on the beam apertures to close the cavity electromagnetically. This is acceptable in a muon channel as a muon interacts only weakly with material in its path. Our approach greatly improves the effectiveness of the cavity, compared with an open-cell design, since the ratio of peak surface field to accelerating field is close to unity in the closed cavity. Pre-curved beryllium windows are used to ensure that their shape is maintained under RF heating. This eliminates the possibility of large frequency changes as the windows flex.

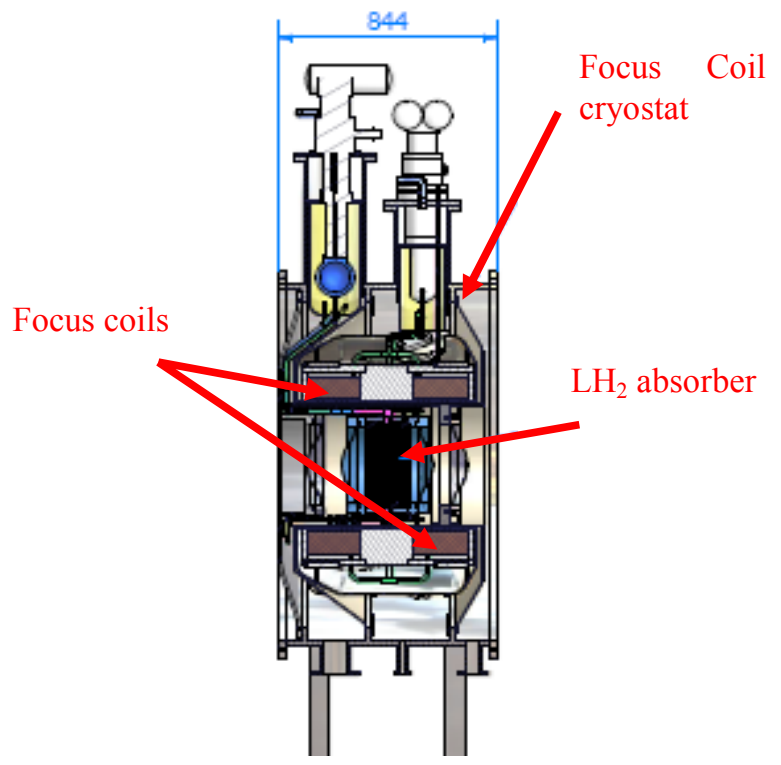

Figure 5: Side view of focus coil module with its $\mathrm{LH}_{2}$ absorber in place. 
To minimize dark currents and breakdown, our cavities will be processed similarly to superconducting cavities. They will be tuned mechanically by altering their shape. A detailed design of the cavity system (see Fig. 6) is under way. It will accommodate the possibility that the cavities may be operated at liquid-nitrogen temperature.

The Coupling Coil is being designed by the Institute of Cryogenics and Superconductivity Technology (ICST) of the Harbin Institute of Technology in China, in collaboration with LBNL. Its design [6] is well along and MICE Collaboration approval to order the superconductor has recently been granted. As seen in Fig. 6, the magnet resides in a separate cryostat and does not share a vacuum enclosure with the RF cavities. The coil will be cooled via 1.5-W pulse-tube cryocoolers. Two coolers are accommodated in the design, but the goal is to be able to operate with only a single cooler.

\section{SPECTROMETER SOLENOIDS}

The spectrometer solenoids [7] are presently being fabricated in industry (Fig. 7). The primary function of this module is to provide a very uniform 4-T field over the region that houses a scintillating fiber tracking detector. Each device has five separate coils - a long central coil, two end coils to ensure uniform field in the tracker region, and two matching coils to ensure proper optical matching into and out of the cooling channel cell.

\section{STATUS AND ANTICIPATED SCHEDULE}

The experiment will be implemented in steps, to make sure there is adequate control of systematic errors. The planned steps and their approximate schedule are shown in Fig. 8. Uncertainty in the first step is due to the solenoid problem discussed earlier.

The detectors required for the first few steps [8] are being fabricated now. The first spectrometer solenoid will be delivered at the end of 2007, with the second following three months later. They will be magnetically measured at Fermilab prior to installation at RAL. Design of the Coupling Coil is complete, and fabrication will begin shortly. Three units will be built, one for the MuCool R\&D program and two for MICE. The bid process for the

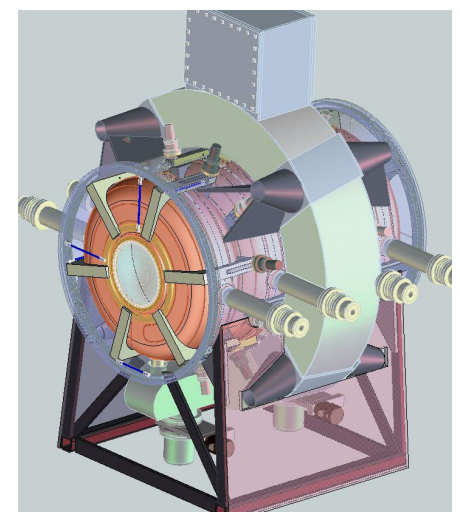

Figure 6: RFCC module showing cavities, 6-spoke tuner mechanism, pumping manifold, and input couplers.

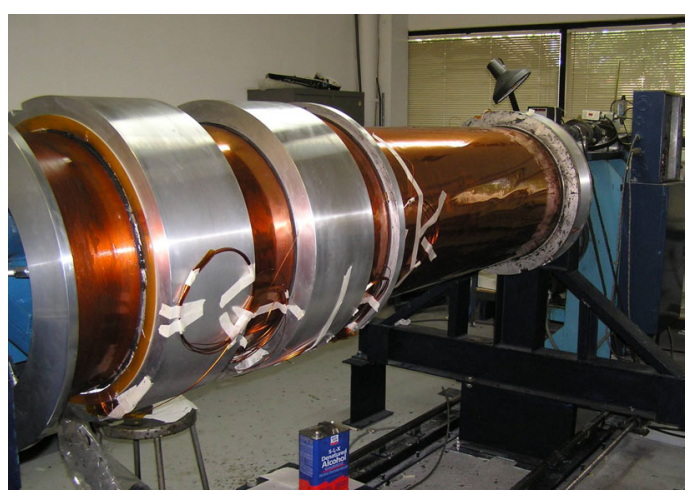

Figure 7: Spectrometer solenoid coils being wound.

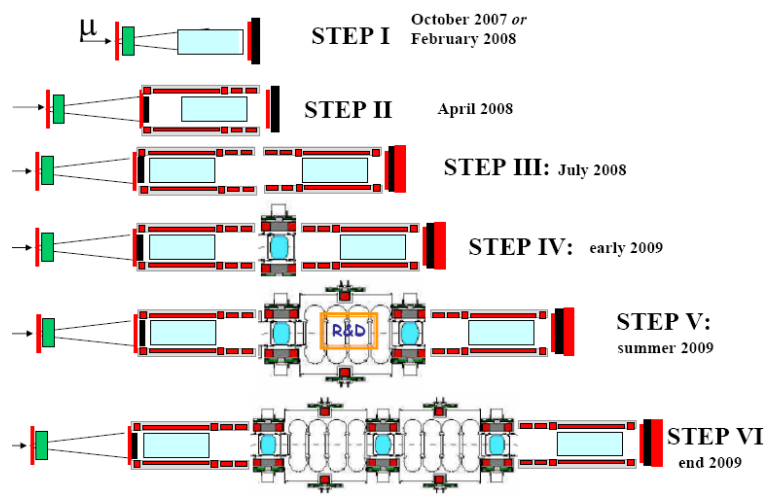

Figure 8: Planned steps for carrying out MICE.

Focus Coil is under way, with responses due later this year. Absorber bodies will be fabricated in Japan, and the thin windows will be built by University of Mississippi.

\section{REFERENCES}

[1] S. Ozaki, R. Palmer, M. Zisman, and J. Gallardo, (eds.), "Feasibility Study II of a Muon-based Neutrino Source," http://www.cap.bnl.gov/mumu/ studyii/final_draft/The-Report.pdf.

[2] M. Cummings and S. Ishimoto, "Progress on the Liquid Hydrogen Absorber for the MICE Cooling Channel," PAC'05, Knoxville, May 2005, p. 1772.

[3] S.Q. Yang et al., "Progress on the Focus Coil for the MICE Channel,” PAC'05, Knoxville, May 2005, p. 3417.

[4] D. Li et al., "Progress on the RF Coupling Coil Module Design for the MICE Channel," PAC'05, Knoxville, May 2005, p. 2869.

[5] R.A. Rimmer et al., "Fabrication of the Prototype 201.25 MHz Cavity for a Muon Ionization Cooling Experiment," PAC'05, Knoxville, May 2005, p. 2080.

[6] M.A. Green and S.P. Virostek, "Progress on the Design and Fabrication of the MICE Coupling Coils," these proceedings.

[7] S.P. Virostek et al., "Progress on the Design and Fabrication of the MICE Spectrometer Solenoids," these proceedings.

[8] T. Hart, "MICE: The International Muon Ionization Cooling Experiment-Phase Space Cooling Measurement," these proceedings. 